\title{
ESEMPLARI DI ARTE APPLICATA CAROLINGIA IN CROAZIA
}

IVO PETRICIOLI

UDC: $745 / 749.033 .4(497.5)$

Review

Manuscript received: 15. 03. 1997.

Revised manuscript accepted: 01. 04. 1997.
I. Petricioli

Dipartimento di storia dell'Arte

Facolta di lettere

Zadar

Croatia

L'Autore descrive alcuni oggetti in metallo, di valore artistico, che risalgono all'epoca dell'impero carolingio e fanno parte del patrimonio culturale croato. Si tratta di quattro paia di speroni e di un incensiere, conservati presso il Museo dei Monumenti Archeologici Croati di Spalato, nonché di due reliquiari appartenenti al tesoro dell'antica cattedrale della città di Nona.

I reperti archeologici e alcuni oggetti di culto rinvenuti testimoniano forti legami culturali tra i Croati e il mondo carolingio. Per il loro numero, al primo posto stanno gli esemplari di armi, rinvenuti durante le ricerche archeologiche nelle tombe paleo-croate. Si tratta di spade forgiate in officine che sorgevano lungo il Reno, di caratteristica forma "carolingia", che secondo la sofisticata analisi degli archeologi specialisti in materia sono state classificate in diversi gruppi e sottogruppi; ci sono poi coltelli da combattimento, lance con "alette" e speroni. Dal punto di vista della storia dell'arte, sono di grande interesse gli speroni che rivelano certi valori stilistici ed estetici della lavorazione orafa. Fra i molti esemplari spiccano quattro paia di speroni rinvenuti nei pressi e all'interno dei resti della chiesa sita nella località di "Crkvina", a Biskupija presso Knin, in cui queste caratteristiche risaltano in modo particolare.

In primo luogo mi soffermerò su un paio di speroni (Fig. 1) di lusso con relativo corredo di affibbiatura della cinghia, rinvenuti in una tomba di pietra con volta all'interno della chiesa. Gli speroni sono di dimensioni piuttosto esigue, da cui si è dedotto che appartenessero a un ragazzo. Sono stati fusi usando una lega di piombo e zinco, con decorazioni a dentello dorate e altre eseguite con la tecnica del niello. La decorazione è composta di piccoli rosoni a petali acuminati che si susseguono in dimensioni più grandi o più piccole lungo l'arco e la punta dello sperone, nonché in alcune parti del corredo per l'affibbiatura. Grazie alla loro ricca decorazione, questi speroni hanno attirato grande attenzione all'epoca del loro ritrovamento, tanto che nel primo articolo pubblicato a riguardo (F. Radić, 1896) si è tentato di riprodurli a colori, con le tecniche grafiche allora disponibili, non ancora sufficientemente sviluppate.

Il secondo paio di speroni, di grandezza normale, è di bronzo fuso e dorato a fuoco (Fig. 2). È stato rinvenuto in un sarcofago costruito con blocchi di pietra che in origine facevano parte di qualche antico edificio monumentale. Le decorazioni sugli speroni sono rifinite a dentello e si distinguono in modo considerevole da quelle che si trovano sugli speroni piccoli appena descritti. I motivi ornamentali consistono in croci, volute, rombi, triangoli e file di granelli eseguite in bassorilievo. A seconda della superficie disponibile i motivi sono disposti e interconnessi in composizioni diverse. Degli speroni sono decorati la punta e un piccolo tratto dell'arco fino alla punta e alla placca a cui erano inchiodate le cinghie, mentre la porzione maggiore dell'ar-

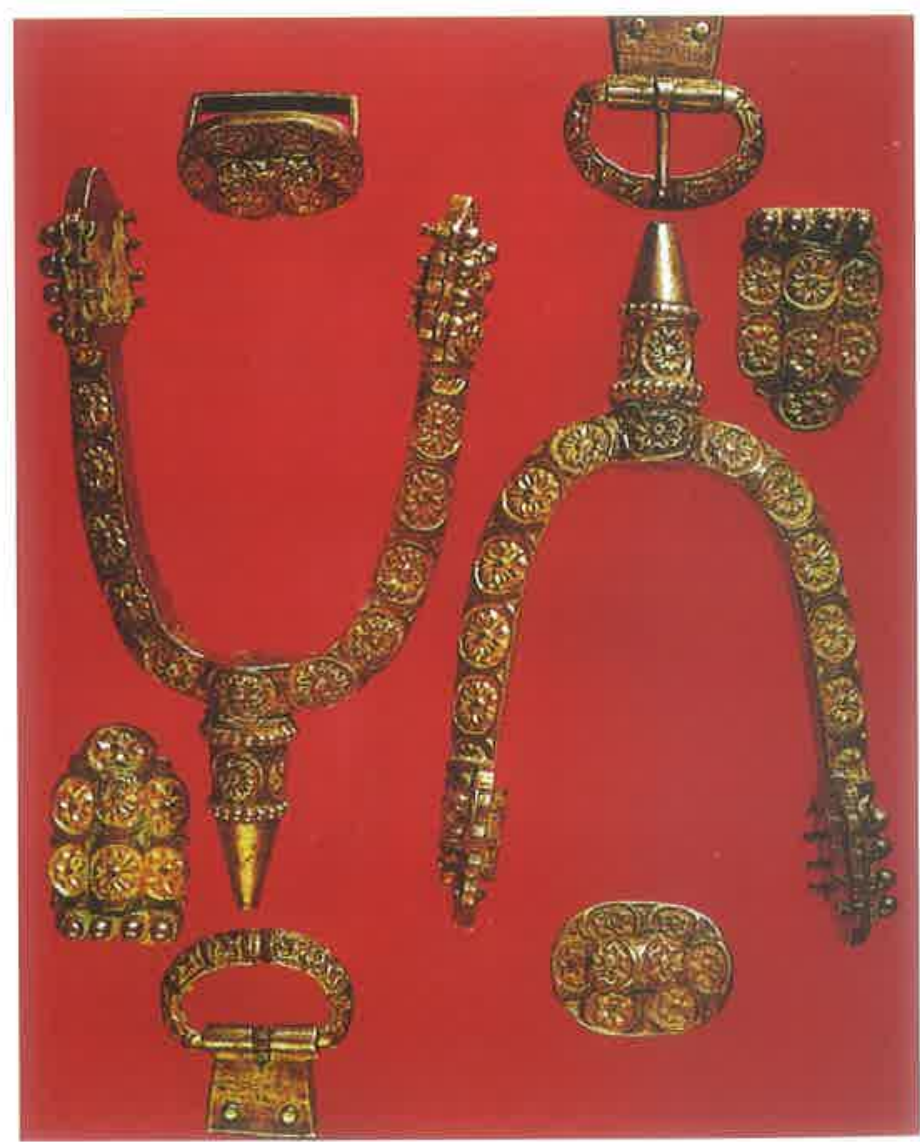

Fig. 1. Gli speroni con rosoni decorativi di Biskupija. 

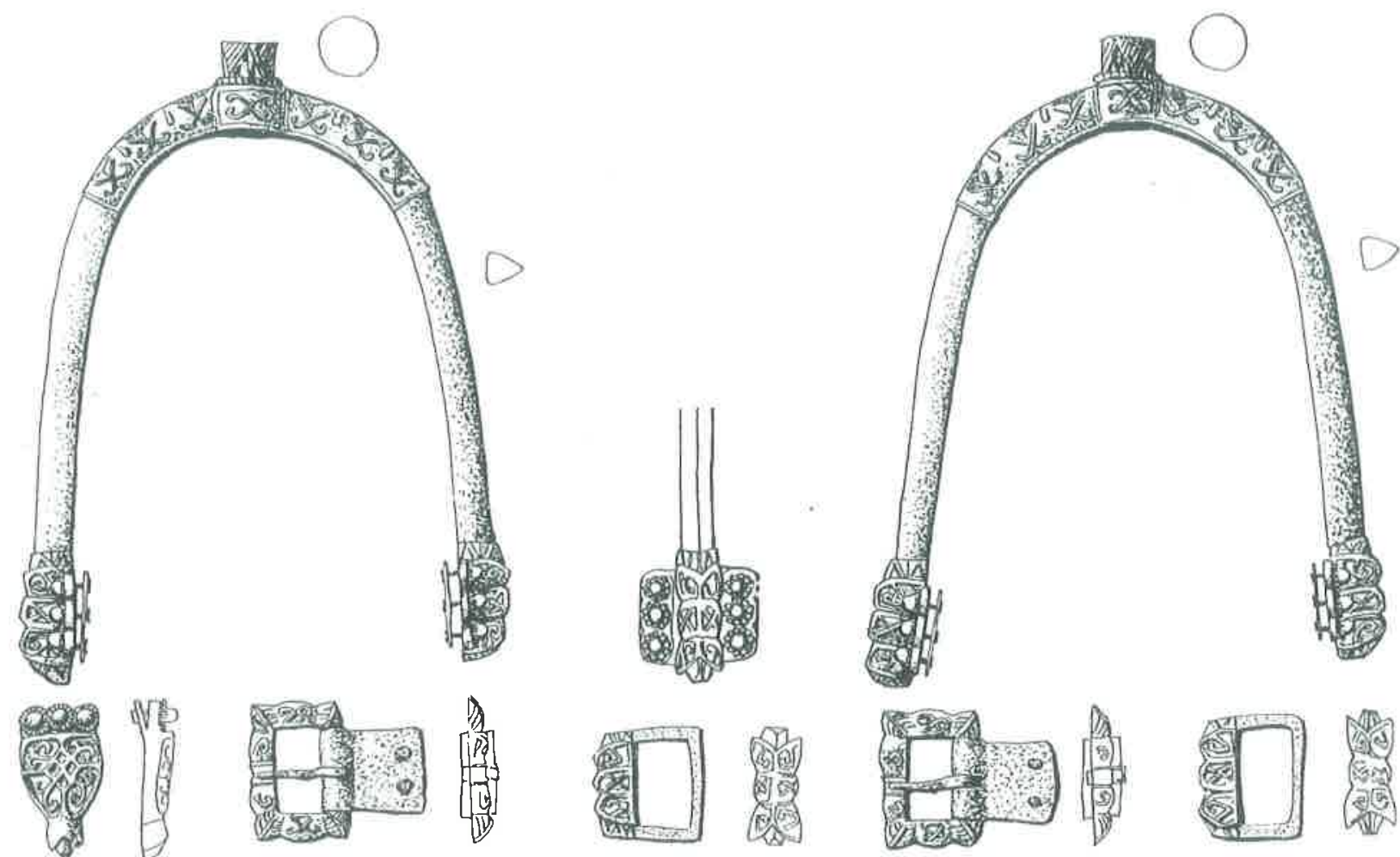

Fig. 3. Gli speroni con decorazione a dentello di Biskupija, terzo esempio (D. Jelovina).

co rimane liscia. Alcune parti del corredo sono riccamente decorate con croci e volute congiunte con molta fantasia in composizioni simmetriche a quattro o otto elementi. Accanto a questi speroni è stato ritrovato un esemplare di moneta dell'imperatore bizantino Basilio I e dei suoi figli (880-886), il quale rinvia alla datazione degli speroni a mo' di terminus ante quem non.

Simili decorazioni che risaltano però in maniera meno evidente, sono visibili sul terzo esemplare proveniente da Biskupija (Fig. 3). Il quarto esemplare consiste in un paio di speroni di bronzo a punta corta, su cui si trova un fregio di sottili volute, eseguito in filo d'argento con tecnica di tassellatura (Fig. 4).

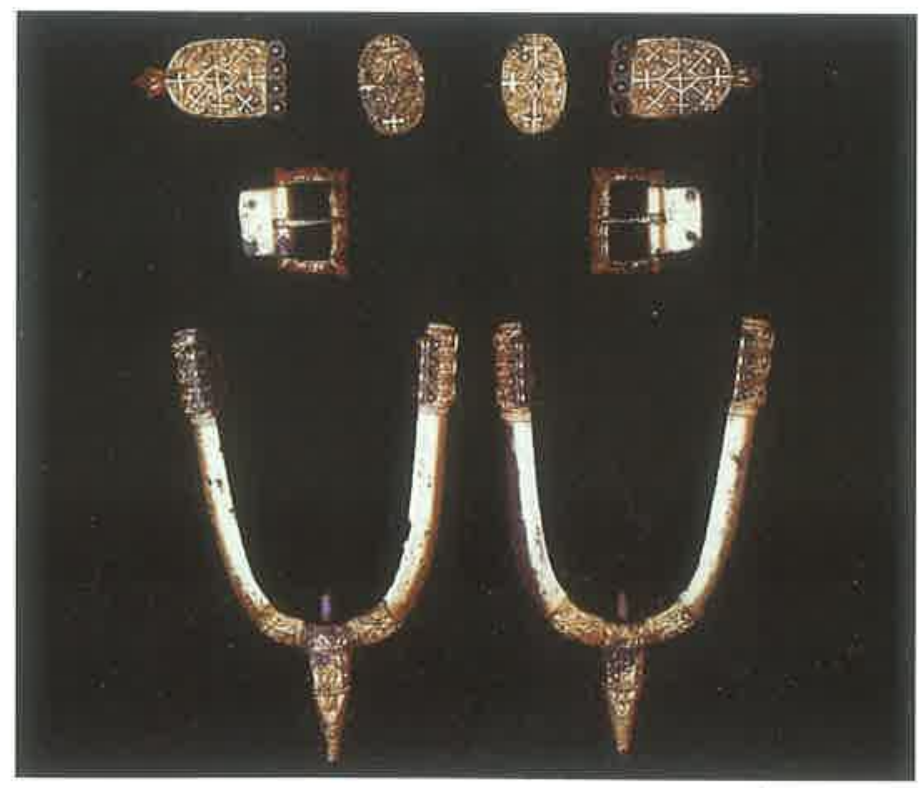

Fig. 2. Gli speroni con motivo ornamentale a croci di Biskupija.
Nei territori costituenti il nucleo dell'impero carolingio non si trovano speroni con decorazioni così ricche. Simili attrezzi sono stati rinvenuti anche nei siti archeologici della Repubblica Ceca, fra i materiali che risalgono all'epoca del regno panmoravo, anche se le loro decorazioni sono diverse da quelle sopra descritte. L'archeologo Z. Vinski prospetta l'ipotesi che gli speroni non fossero d'importazione, ma prodotti da artigiani locali sotto l'influenza dell'arte carolingia.

Di particolare valore è l'incensiere (Fig. 5) custodito insieme agli speroni in oggetto presso il Museo dei Monumenti Archeologici Croati di Spalato. L'incensiere è giunto al museo nel 1925 dal villaggio di Zetina, nei pressi della chiesa paleo-croata di S. Salvatore; nessuno se n'è occupato fino al saggio scientifico pubblicato nel 1958 da Ksenija Vinski-Gasparini. In quell'occasione l'incensiere venne ripulito e sottoposto a trattamenti conservativi, per cui fu possibile analizzarne anche la tecnica di fabbricazione. L'incensiere è in argento fuso, mentre le decorazioni sono lavorate a dentello e niello, e sono dorate. L'oggetto è di dimensioni modeste: è alto $6,2 \mathrm{~cm}$ e delle stesse dimensioni è anche il diametro del recipiente. Quest'ultimo è oviforme, ed è alto 4,5 $\mathrm{cm}$. Il piccolo piedistallo è anch'esso rotondo, concavo verso l'interno, mentre in basso si incurva e si allarga leggermente. Il diametro della base è di $3,8 \mathrm{~cm}$. All'interno del recipiente se ne trova un altro, la cosiddetta concula, in rame lavorato a sbalzo. La decorazione sulla superficie esterna del recipiente è suddivisa in tre bande, mentre sul bordo superiore e sul raccordo con il piedistallo si trova una fila di granelli. La banda superiore è composta di due file di rombi incavati ed è dorata. La fascia centrale, la principale, è ornata di quattro arcate, anch'esse dorate e decorate con rombi incavati. Le superfici tra le arcate portano ciascuna, graffito, un fiore di giglio stilizzato. La banda inferiore è 

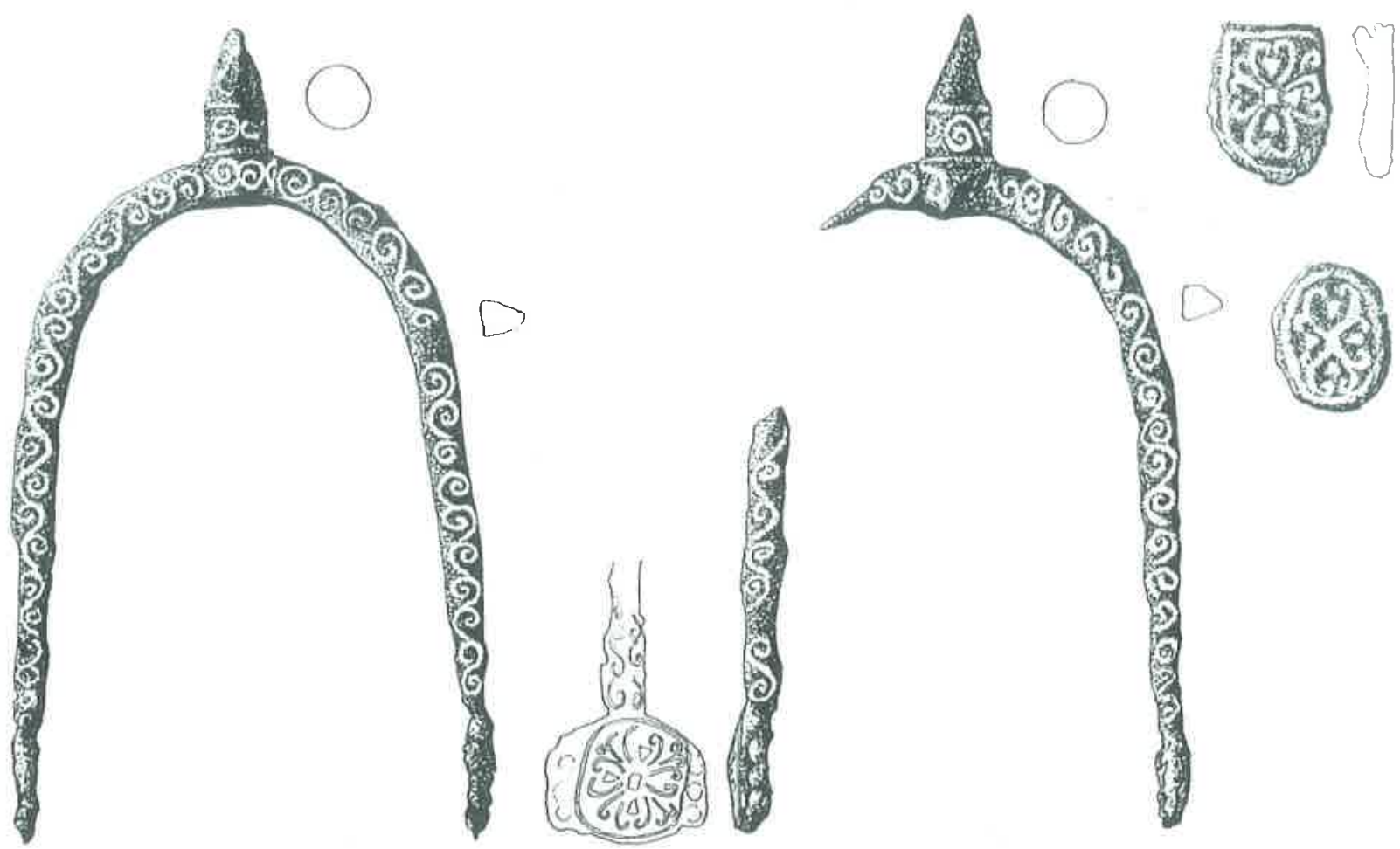

Fig. 4. Gli speroni con decorazione a tassello di Biskupija, quarto esempio (D. Jelovina).

simile alla superiore. Il piccolo piedistallo è suddiviso in tre fasce ristrette. Sulla superficie della fascia superiore e di quella inferiore, entrambe dorate, sono graffiti dei triangoli, mentre la banda centrale è priva di decorazioni. L'orlo del piedistallo reca una fila di granelli assai più piccoli di quelli che si trovano sul recipiente. All'interno del piedistallo è inchiodato un vasetto rovesciato, in rame dorato. Le tre catene a cui è appeso l'incensiere sono di filo intrecciato e lunghe $28 \mathrm{~cm}$. Sono attaccate a un supporto dorato a tre bracci orizzontali che terminano con teste stilizzate di animali dai grandi occhi lavorati a dentello. La parte verticale del supporto ha la forma di due coni tronchi. È ornata di file di granelli e di triangoli graffiti e dorati. Sopra di essa è fissato il gancio. Incluso il gancio, il supporto è alto 8,3 $\mathrm{cm}$, mentre i bracci sono lunghi $2,3 \mathrm{~cm}$.

K. Vinski-Gasparini si è premurata di cercare del materiale comparativo nella bibliografia disponibile, senza trovare tuttavia neanche un incensiere affine a questo. Le somiglianze maggiori sono quelle con il noto calice del duca bavarese Tassilo di Kremsmünster, datato prima del 788. Il calice è stato realizzato mediante la stessa tecnica orafa dell'incensiere, ma il carattere degli ornamenti è diverso. Qui si presentano motivi cosiddetti insulari, motivi a intreccio di animali, di origine irlandese-anglosassone, lavorati in maniera assai più elaborata che non nel nostro incensiere. La lavorazione degli ornamenti dell'incensiere può essere paragonata alle fibule germaniche, per cui K. Vinski-Gasparini giunge alla conclusione che l'incensiere in questione poteva essere stato realizzato in officine lungo il Reno e portato in Croazia da missionari franchi. L'autrice reputa che possa essere stato realizzato verso la metà dell'VIII secolo.

Nella chiesa parrocchiale di Nona è oggi conservato il tesoro dell'ex cattedrale della città. Fra i numerosi reliquiari medievali di valore, ne spiccano due, la cui data di realiz- zazione, indicata nella bibliografia, viene situata in età carolingia; si ritiene che i reliquiari siano giunti a Nona tramite missionari franchi al tempo della diffusione del cristianesimo fra i croati.

Mi soffermerò dapprima sul reliquiario-scrignetto di un santo ignoto (Fig. 6). Lo scrignetto è in legno, rivestito di lamina d'argento. È lungo $24,5 \mathrm{~cm}$, largo 10 e alto $12 \mathrm{~cm}$. Ha forma di sarcofago con tetto a doppio spiovente; nella parte inferiore reca inchiodati quattro piccoli piedini in rame. Il coperchio è composto di due placche collegate da cerniere, che lo rendono flessibile. Tutti i lati dello scrignetto, tranne uno dei lunghi, sono decorati con rosoni lavorati a sbalzo, tre dei quali sono applicati sui lati lunghi e sul coperchio, mentre sui lati corti ve n'è applicato uno solo. Si ripetono quattro tipi di rosoni, due a sei foglie e due a otto foglie. Uno di quelli a sei foglie e uno di quelli a

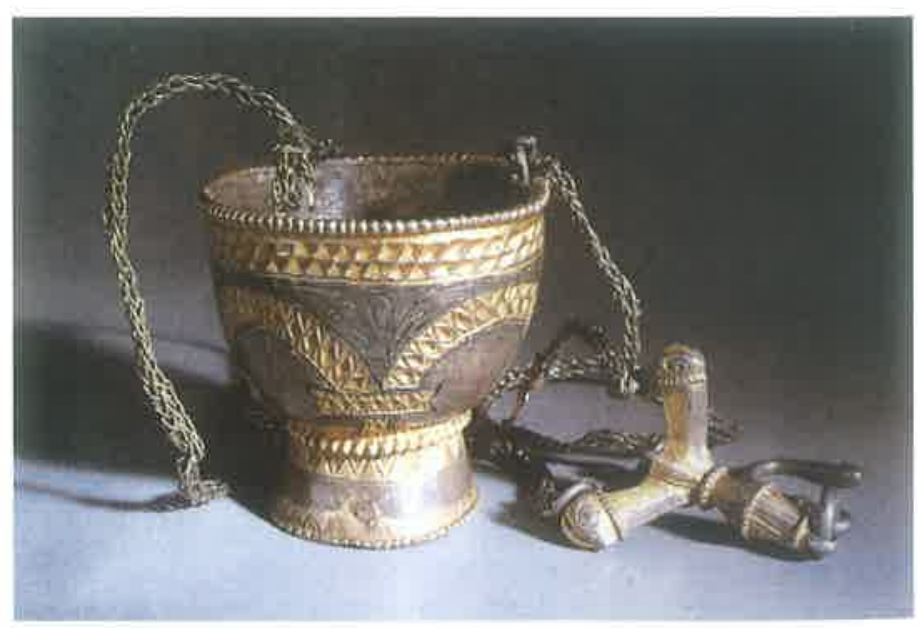

Fig. 5. L'incensiere di Stara Vrlika. 


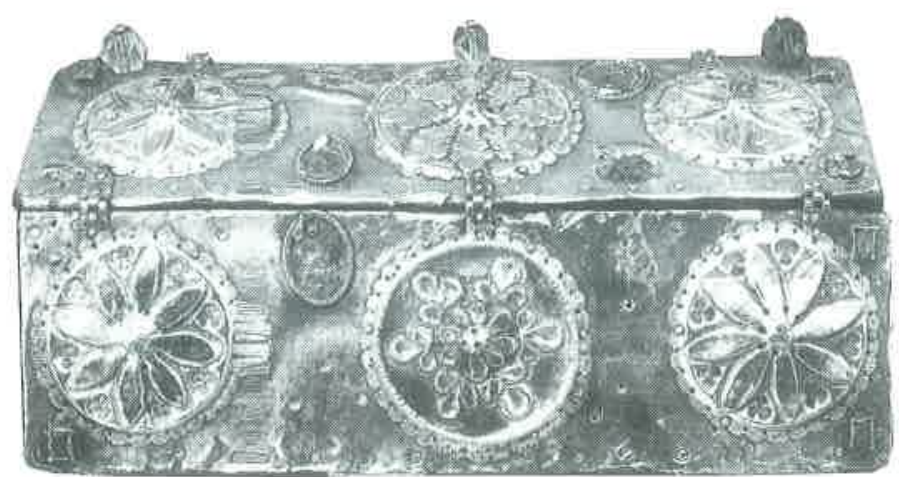

Fig. 6. Il reliquiario-scrignetto dal tesoro della chiesa di Nona.

otto foglie hanno i petali scissi, mentre gli altri sono composti di petali semplici tracciati con il compasso. Su uno dei lati corti si trova un esagono con fiore tracciato con il compasso. Vi sono applicate o, per meglio dire, vi erano applicate delle gemme, probabilmente false, in pasta di vetro. Ve n'erano dodici in tutto, quelle ovali, più grandi, tra i rosoni, delle quali non rimane traccia e che spesso mancano anche dell'incastonatura d'argento. Le piccole gemme quadrate si trovavano agli angoli dei lati lunghi. Ne sono rimaste solo tre, mentre sul dorso del coperchio vi sono applicati tre cristalli.

L'ornamento primitivo e le gemme applicate indicano l'Alto Medioevo come periodo di realizzazione di questo reliquiario. Jelić lo fa risalire al VII od VIII secolo. Rosoni tracciati con il compasso, ma graffiti, si trovano sul noto reliquiario di Saint Benoît sur Loire, dell'Alto Medioevo, commissionato da Mumma e che si fa risalire al VII secolo. I rosoni del reliquiario di Nona sono plastici e lavorati da una mano più abile. Non è possibile stabilire una datazione più precisa, poiché l'ornamento primitivo consente di variare quest'ultima nel raggio di alcuni secoli, mentre non esistono dati storici su cui basare le stime.

L'altro reliquiario in questione è ben noto nella bibliografia nostrana e ne sono state date frequenti riproduzioni. Si tratta del reliquiario di S. Anselmo, patrono di Nona (Fig. 7); ha la forma a borsetta (ted. Bursereliquiar), caratteristica dei reliquiari dell'Alto Medio Evo. Reliquiari di questo tipo si trovano sia nell'epoca merovingia che in quella carolingia. Alcuni esemplari conservati in Francia e in Germania sono più sfarzosi di quello di Nona e di solito sono ornati con gemme, ma ciò nonostante i paragoni sono egualmente possibili. Il reliquiario in questione è alto $34,5 \mathrm{~cm}$, largo alla base $20,5 \mathrm{~cm}$, alla sommità $17 \mathrm{~cm}$, e nella parte più stretta $13 \mathrm{~cm}$; è spesso $5 \mathrm{~cm}$ alla base e $1 \mathrm{~cm}$ alla sommità. È decorato esclusivamente a sbalzo in lamina d'argento dorata. Già a prima vista si nota che le placche originali sono state danneggiate e che il reliquiario è stato successivamente riparato. Anche se la composizione principale, con le figure di santi sul davanti, ha subìto i danni più gravi nella parte bassa, risulta comunque facile evocarne l'aspetto originale. Sotto ciascuna delle tre arcate è posta una figura di santo. Le arcate sono irregolari, di altezza uguale, ma di larghezza diversa: quella a sinistra è la più larga, quella a destrá la più stretta. Sotto l'arcata centrale è effigiata una santa in atto di adorazione, sotto quella a sinistra un santo orante, di aspetto giovanile e con la tonsura, mentre sotto quella a destra, purtroppo assai danneggiato, sta un santo

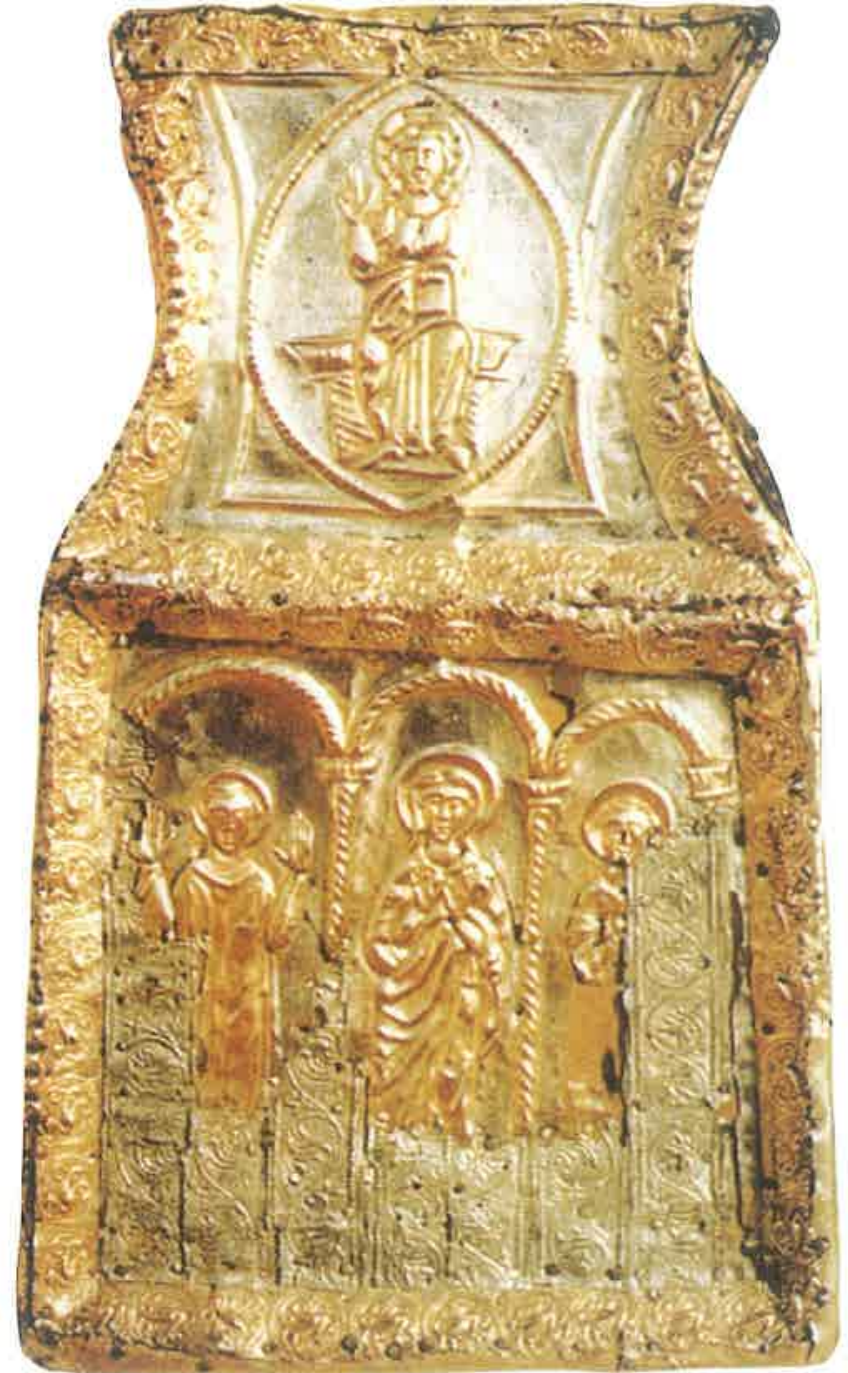

Fig. 7. Il reliquiario a forma di "bursa" (borsetta) dal tesoro di Nona.

di aspetto giovanile che reca nella mano destra un incensiere. Senza dubbio vi sono rappresentati i santi patroni della città di Nona: Marcella, Ambrosio e Anselmo. Nella parte superiore vi è rappresentato il Cristo sul trono all'interno della mandorla mistica. I volti delle figure sono oblunghi, leggermente a forma di pera, le mani sono allungate e modellate morbidamente. I panneggi dei vestiti sono semplici, ma ben definiti nello stesso spessore di rilievo riscontrabile per le figure e le aureole. È evidente la "calligrafia" dell'orafo. Benché la tecnica di lavorazione a sbalzo di questo reliquiario sia più semplice che non negli esempi rappresentativi dell'oreficeria carolingia, trattati "pittoricamente" con la stessa tecnica, come ad es. il Codex aureus di San Emerano, le proporzioni dei volti e soprattutto la modellatura delle mani, indicano un'affinità con le figure lavorate a sbalzo del tardo periodo merovingio, nonché di quello carolingio. Il lato posteriore del reliquiario è decorato in maniera molto primitiva. Attraverso il piano centrale e la parte superiore sono tracciate delle diagonali con file di piccoli emisferi sbalzati, tra cui si trovano motivi circolari. Nella parte superiore, quattro cerchi più piccoli sono ricoperti due a due dallo stesso ornamento primitivo - circoli concentrici o porzioni di circoli. Sul piano centrale i cerchi sono assai più grandi e la loro decorazione è a forma di croce. Anche la bordatura di queste due superfici è lavo- 
rata in maniera assai primitiva. Le altre parti del reliquiario non sono originali, ma risalgono a un restauro avvenuto molto più tardi. La superficie anteriore, laddove le figure erano danneggiate, è stata integrata usando una nuova lamina d'argento su cui sono lavorati a sbalzo motivi di viticcio, tipici delle realizzazioni orafe duecentesche e trecentesche. I piani anteriori sono orlati di un nastro d'argento dorato, su cui viene ripetuto a più riprese il motivo lavorato a sbalzo mediante lo stesso stampo. Il motivo consiste in un cerchio che inscrive un fantasioso drago, il quale rappresenta una strana combinazione di leone, uccello e serpente. Lo stampo era di buona fattura e anche la realizzazio- ne è di alto livello artigianale. Le superfici laterali del reliquiario sono trattate diversamente, con vari motivi vegetali lavorati a sbalzo, ma in maniera assai primitiva.

Nella bibliografia in cui questo reliquiario è stato finora menzionato, esso viene legato alla conversione al cristianesimo dei croati operata dai missionari franchi ai tempi di Carlo Magno, per cui anche la provenienza del reliquiario viene fatta risalire alle officine franche dell'epoca. La realizzazione del reliquiario, però, sarebbe potuta avvenire nel periodo della fondazione della diocesi croata di Nona, il che sposterebbe la sua datazione qualche decennio più tardi, ma comunque nell'ambito della cultura carolingia.

\section{BIBLIOGRAFIA ESSENZIALE}

S. GUNJAČA, D. JELOVINA, Starohruatska baština [Patrimonio paleo-croato], Zagabria 1976

L. JELIĆ, Thaesaurus ecclesiae cathedralis nonensis, Compte-rendu du Quatrième Congrès Scientifique International des Catholiques tenu à Fribourg 1897, Fribourg 1898

D. JELOVINA, Starohrvatske nekropole na području između rijeka Zrmanje i Cetine [Le necropoli paleo-croate nella zona tra i fiumi Zermagna e Zetina], Spalato 1976

D. JELOVINA, Mačevi i ostruge karolinškog obilježja u Muzeju hrvatskih arheoloških spomenika u Splitu [Spade e speroni di carattere carolingio presso il Museo di Monumenti Archeologici Croati di Spalato], Spalato 1986

LJ. KARAMAN, Iz kolijevke hrvatske prošlosti [Dalla culla del passato croato], Zagabria 1930

LJ. KARAMAN, Živa starina [Antichità viva], Zagabria 1943

LJ. KARAMAN, O porijeklu starohrvatskih ostruga [Sull'origine degli speroni paleo-croati], Starohrvatska prosvjeta, serie III, fascicolo 4 , Zagabria 1955 I. PETRICIOLI, Osvit na ninske građevine i umjetničke spomenike srednjega i novoga vijeka [Considerazioni sugli edifici e sui monumenti artistici di Nona, dal Medio Evo all'epoca moderna], Radovi Instituta JAZU u Zadru, fascicolo 16-17, Zara 1969

F. RADIĆ, Srebrene ostruge i saponi iz starohrvatskih grobova u Biskupiji kod Knina [Speroni d'argento e pastoie nelle tombe paleo-croate a Biskupija presso Knin], Starohrvatska prosvjeta, a. II, n. 1, Knin 1896

F. RADIĆ, Grobna raka iz starohrvatske biskupske bazilike su. Marije u Biskupiji kod Knina [La tomba sepolcrale nella basilica episcopale paleo-croata di S. Maria a Biskupija presso Knin], Starohrvatska prosvjeta, a. II, n. 2, Knin 1896

K. VINSKI-GASPARINI, Kadionica iz Stare Vrlike [L'incensiere di Stara Vrlika], Starohrvatska prosujeta, serie III, fascicolo 6, Zagabria 1958

Z. VINSKI, Oružje na području starohrvatske države do god. 1000. [Le armi nel territorio dello Stato paleo-croato fino all'anno Mille], I Miedzynardowy kongres archeologii slowianskiej (1965), T. III, Varsavia 1970

\section{PRIMJERCI KAROLINŠKOG UMJETNIČKOG OBRTA U HRVATSKOJ}

\section{SAŽETAK}

Metalnih predmeta umjetničke vrijednosti iz razdoblja karolinške kulture sačuvao se u Hrvatskoj mali broj, ali oni zaslužuju zbog svojih specifičnih osobina nemalu pozornost.

Najstariji predmet koji se datira oko polovice VIII. st. jest mala kadionica pronađena u blizini poznate starohrvatske crkve sv. Spasa na vrelu Cetine. Izrađena je od srebra, lijevana je i ukrašena jednostavnim geometrijskim motivima u tehnici rovašenja i niella, djelomično pozlaćenim. Sačuvao se i lanac okačen za trostruki nosač s kukom. Po načinu ukrašavanja podsjeća na poznati kalež bavarskog vojvode Tasila, ali je znatno skromnijeg izgleda i zanatske obrade. Čuva se u Muzeju hrv. arheol. spomenika u Splitu.

U riznici župne crkve u Ninu, nekadašnje katedrale, čuvaju se dva relikvijara, koji se po svojim osobinama mogu datirati u karolinško doba. Jedan je četvrtasta škrinjica s poklopcem poput dvoslivnog krova ukrašena primitivno izrađenim i pozlaćenim rozetama, a drugi u karakterističnom obliku "burse" (torbice), uobičajenom u karolinškom zlatarstvu, s iskucanim reljefnim svetačkim likovima, koji po stilizaciji anatomskih detalja i draperije podsjećaju na slična karolinška ostvarenja.

Među umjetnički obrađene predmete treba svrstati i četiri para ostruga pronađenih u grobovima u ruševinama trobrodne crkve sv. Marije u Biskupiji kod Knina, a čuvaju se u Muzeju hrv. arheol. spomenika u Splitu. Lijevane su, a ukrašene raznim motivima izvedenim u raznim tehnikama. Tri para imaju rovašene ornamente i pozlaćene su, a jedan je ukrašen tauširanjem. 
